\title{
Authors' reply to Dr. Sabour
}

\author{
T. B. Mikkelsen ${ }^{1}$ (D) $\cdot$ B. Sørensen ${ }^{2} \cdot$ K. B. Dieperink ${ }^{1,3}$
}

Received: 20 March 2017 / Accepted: 24 April 2017 /Published online: 2 May 2017

(C) Springer-Verlag Berlin Heidelberg 2017

\section{Authors' response}

We appreciate the interest of Dr. Sabour in our recent published article "Prediction of rehabilitation needs after treatment of Cervical Cancer. What do late adverse effects tell us?" and we read his comments with interest. We can see from Dr. Sabour's publications that he is interested in methodological issues in multiple research fields with more than 90 commentary papers published, including a high number of self-references.

Dr. Sabour expressed concern that the paper stated misleading results because we did not complete a prediction study with two separate cohorts.

However, we believe that Dr. Sabour's comments relate to the use of the word "prediction" in the title of our article. This is a common English word that, according to dictionary definition, means what someone thinks will happen or an informed guess. We sought to write an article that was relevant to clinical practice and chose the word "prediction" as a word to describe how the results could be used to identify cervical cancer patients who could need rehabilitation and not as a statistical term.

Unfortunately, Dr. Sabour assessed the article as a prediction study, even though it was not described as such in either the purpose or methods sections. In the conclusion, we did not write that we had developed a prediction model, but suggested that young, obese survivors with locally advanced cervical cancer and survivors who received chemotherapy may be at serious risk of developing late adverse effects; thus, rehabilitation should target these needs.

We do, however, agree that the word "prediction" could be misunderstood in this context and we will be more careful about the use of the word "prediction" in the future.

\section{Compliance with ethical standards}

Conflict of interest The authors declare that they have no conflict of interest.
T. B. Mikkelsen

Tina.broby.mikkelsen@rsyd.dk

1 Knowledge Centre for Rehabilitation and Palliative Care, Vestergade 17, 5800 Nyborg, Denmark

2 Department of Oncology, Lillebaelt Hospital Vejle, Kabbeltoft 25, 7100 Vejle, Denmark

3 Department of Oncology, Odense University Hospital, Sdr. Boulevard 29, Indgang 99, 2 Sal, 5000 Odense C, Denmark 